\title{
Effects of NO Synthase Inhibitors on the Synovial Microcirculation in the Mouse Knee Joint
}

\author{
A. Veihelmann ${ }^{a, b}$ F. Krombach ${ }^{b}$ H.J. Refior ${ }^{a}$ K. Messmer ${ }^{b}$ \\ aDepartment of Orthopedics and bInstitute for Surgical Research, Ludwig Maximilians University of Munich, \\ Germany
}

\section{Key Words}

Nitric oxide · Synovial microcirculation · L-NAME .

L-NIL · Knee joint · Mouse · Arthritis

\begin{abstract}
Production of nitric oxide by the inducible NO synthase (iNOS) is known to be enhanced in chronic joint inflammation and osteoarthritis as well as aseptic loosening of joint prostheses. Initial studies yielded promising results after inhibition of the nitric oxide synthase (NOS). However, the effect of NOS inhibition has not been studied at the site of the primary function of NO, the microcirculation of the synovium in vivo. Using our recently developed model for the in vivo study of synovial microcirculation in the mouse knee joint, the effects of selective versus nonselective inhibition of iNOS were investigated by means of intravital fluorescence microscopy. After resection of the patella tendon, the synovial fatty tissue was exposed for intravital microscopy. Diameter of arterioles, functional capillary density (FCD), diameter of venules, venular red blood cell velocity and leukocyte-endothelial cell interaction were quantitatively analyzed before, and 10 and $60 \mathrm{~min}$ after intravenous injection of NOS inhibitors [selective iNOS inhibitor $\mathrm{N}$-iminoethyl-Llysine (L-NIL), and nonselective NOS inhibitor $\mathrm{N}^{\mathrm{G}}$-nitro$L$-arginine methyl ester (L-NAME)]. Our results demonstrate that L-NAME causes a significant decrease in the arteriolar diameter and FCD associated with an increase
\end{abstract}

\begin{tabular}{ll}
\hline KARGER & ( 1999 S. Karger AG, Basel \\
Fax +4161306 1234 & \\
$\begin{array}{l}\text { E-Mail karger@karger.ch } \\
\text { www.karger.com }\end{array}$ & $\begin{array}{l}\text { Accessible online at: } \\
\text { www.karger.com/journals/jvr }\end{array}$
\end{tabular}

in the leukocyte accumulation in the synovium in vivo. In contrast, L-NIL neither altered the microhemodynamics nor the leukocyte-endothelial cell interaction in the synovium, indicating its potential use for selective inhibition of iNOS in joint inflammation. Using our method, further studies will provide new insights into the unknown effect of NOS inhibition on the synovial microvasculature in inflammatory joint disease in vivo.

Copyright $\odot 1999$ S. Karger AG, Basel

\section{Introduction}

Many inflammatory diseases including chronic joint inflammation are associated with an enhanced production of nitric oxide. A variety of different types of cells are able to synthesize NO from arginine in a reaction catalyzed by NO synthase (NOS). This enzyme exists in the form of several isoenzymes, the major distinction being between inducible (iNOS) and constitutive forms (cNOS). Cells which contain cNOS synthetize rapidly, but transiently, small amounts of NO. In contrast, cells expressing iNOS synthetize large amounts of NO for an extended period following a delay of several hours during which the enzyme is induced.

The findings that articular chondrocytes release large amounts of NO by iNOS after incubation with proinflammatory cytokines or endotoxin and that also cells of the synovium synthetize NO via iNOS in the arthritic joint

Dr. Andreas Veihelmann

Department of Orthopedics, Ludwig Maximilians University of Munich Marchioninistrasse 15

D-81377 Munich (Germany)

Tel. +49 897095 4355, Fax +49 897095 8897, E-Mail andyvei@lrz-muenchen.de 
provided the first evidence that NO may be involved in the pathogenesis of joint disease [1,2]. Subsequent animal studies confirmed these observations by showing that chondrocytes and synovial fibroblasts as well as extravasated neutrophils and macrophages express iNOS in chronic inflammatory joint diseases [2, 3]. In patients with joint inflammation, induction of iNOS was demonstrated in neutrophils, synovial fibroblasts, and chondrocytes $[4,5]$. Furthermore, NO production has been shown to be involved in osteoarthritis, ligament healing, and aseptic loosening of endoprostheses [6]. These and other findings envisage a potential use of therapeutic inhibition of NO synthesis in joint inflammation. Nevertheless, the impact of the enhanced NO release by iNOS in inflammation on the course of the disease has been discussed controversially. Studies with the use of nonselective NOS inhibitors showed an improvement in liver detoxification in rats with inflammatory liver dysfunction [7], as well as a suppression of arthritis in rats [8]. Investigations using selective iNOS inhibitors in experimental arthritis reported contradictory results $[9,10]$. Furthermore, there are several studies suggesting even beneficial effects of selective iNOS inhibition in chronic joint inflammation $[6,2,10]$. On the other hand, serious side effects of nonselective NOS inhibition on macro- and microhemodynamics have been reported [11]. Recently, it has been shown that inhibition of $\mathrm{NO}$ synthesis with $\mathrm{N}^{\mathrm{G}}$-nitro- $L$-arginine methyl ester (L-NAME) resulted in an enhanced leukocyte-endothelial cell interaction and in alterations of the endothelial cytoskeleton with subsequent albumin leakage from mesenteric postcapillary venules [12-14]. Interestingly, in single perfused frog mesenteric venular vessels nonselective inhibition of NOS led to a decrease in microvessel permeability $[15,16]$. However, some authors postulated that iNOS prevents leukocyte adherence in intestinal inflammation and endotoxemia $[17,18]$.

Most of the investigations regarding NOS inhibition and its effects on the microcirculation have used the rat mesenterium. Yet, no studies have been reported which directly demonstrate the in vivo effects of selective versus nonselective NOS inhibitors at the site of the synovial microcirculation. Therefore, it was our aim to investigate the in vivo changes of the synovial microcirculation and the leukocyte-endothelial cell interactions in the mouse knee joint after selective, N-iminoethyl- $L$-lysine (L-NIL) [19], versus nonselective NO synthase inhibition (LNAME), using our recently developed model for intravital microscopic analysis of the mouse synovial microvasculature [20].

\section{Materials and Methods}

\section{Microsurgical Procedure}

Female Balb/c mice (Charles River, Sulzfeld, Germany), weighing 18-21 g, were used for the experiments. The animals were kept in an air-conditioned environment with 12-hour light/dark cycles, housed in polystyrene cages and fed laboratory chow, supplemented with $120 \mathrm{mg}$ of vitamin $\mathrm{E}$ and 18,000 IU of vitamin A per kilogram body weight (ssniff, Spezialdiäten, Soest, Germany), and water ad libitum. Anesthesia was performed by inhalation of isoflurane 1.2\% (Forene, Abbott, Wiesbaden, Germany) and a combination of $\mathrm{O}_{2} /$ $\mathrm{N}_{2} \mathrm{O}$. The mean arterial blood pressure was assessed by an arterial catheter that was inserted into the tail artery and connected to a pressure transducer (Sirecust 300 D, Siemens, Erlangen, Germany). The microsurgical procedure was performed as described previously [20]. Animals were kept on a heating pad to stabilize body temperature which was controlled by a rectal probe. The left limb was placed on a stage with a slight flexion of the knee joint. Immobilization of the extremity was achieved by fixation in silicone (Classic M, Munich, Germany). After a 1-cm incision was made distally of the patella tendon, partial skin resection ensued. The patella tendon was carefully mobilized and partially resected. Afterwards, the 'Hoffa's fatty body' (intra-articular synovial tissue of the knee joint) was visualized without the need of opening the joint capsule. After superfusion with $5 \mathrm{ml}$ of sterile saline, a cover glass was placed on the knee capsule, and the intravital microscope was directed on the synovium. Special care had to be taken to avoid any constraint of the preparation. After intravital microscopy, the animals were sacrificed with $10 \mathrm{mg}$ of pentobarbital (Nembutal; Sanofi, Hannover, Germany) intravenously.

\section{Experimental Protocol}

The animals were allocated into three groups. The control group received a bolus injection of $0.1 \mathrm{ml}$ sterile saline $(\mathrm{n}=11)$ through a venous catheter. In two other groups, either the nonselective NOS inhibitor L-NAME ( $5 \mathrm{mg} /$ animal, $\mathrm{n}=7$ ) or the selective iNOS inhibitor NIL ( $2 \mathrm{mg} / \mathrm{animal}, \mathrm{n}=7)$ was administered intravenously as a bolus immediately after the preparation. The dose of L-NIL has been chosen according to results of pilot studies in 3 mice with an inflamed knee joint. In these experiments, $2 \mathrm{mg}$ of L-NIL given as a bolus intravenously were found to completely abolish elevated plasma nitrite/nitrate concentrations to almost control levels. Intravital microscopy was performed in all animals prior to as well as 10 and $60 \mathrm{~min}$ after the administration of the inhibitors.

\section{Intravital Fluorescence Microscopy}

The microscopic setup has been described in detail previously [21]. A 20-fold water immersion objective (Zeiss Axiotech vario 100 HD microscope, Acroplan $20 \times / 0.5 \mathrm{~W}$, Zeiss, Oberkochen, Germany) was used to observe the synovial microvasculature under epiillumination. The images were recorded with a video camera (FK 6990 IQ-S, Piper, Schwerte, Germany) onto S-VHS video tapes (Sony, Köln, Germany) for later off-line analysis. Epi-illumination was achieved with either a 12-volt, 100-watt halogen lamp, or a 100watt HBO mercury vapor lamp (Zeiss), depending on the fluorescent marker. The halogen lamp was used in conjunction with the Zeiss filter set 09 (BP 450-490, FT 510, LP 520) for measurements involving fluorescein isothiocyanate-labeled dextran (FITC-dextran). The HBO lamp and the Zeiss filter set 15 (BP 450-490, FT 546/12, FT 580, LP 590) were used for measurements involving rhodamine $6 \mathrm{G}$. Two to three regions of interest per animal were measured, contain- 
Table 1. Macro- and microhemodynamic parameters

\begin{tabular}{|c|c|c|c|c|c|c|c|c|c|}
\hline \multirow[t]{2}{*}{ Parameter } & \multicolumn{3}{|c|}{ Control $(n=11)$} & \multicolumn{3}{|c|}{ L-NAME $(\mathrm{n}=7)$} & \multicolumn{3}{|c|}{ L-NIL (n = 7) } \\
\hline & baseline & $10 \mathrm{~min}$ & $60 \mathrm{~min}$ & baseline & $10 \mathrm{~min}$ & $60 \mathrm{~min}$ & baselline & $10 \min$ & $60 \mathrm{~min}$ \\
\hline MAP, $\mathrm{mm} \mathrm{Hg}$ & $78 \pm 7$ & $84 \pm 7$ & $85 \pm 8$ & $80 \pm 7$ & $89 \pm 13$ & $87 \pm 10$ & $80 \pm 7$ & $84 \pm 8$ & $82 \pm 5$ \\
\hline Arteriole diameter, $\mu \mathrm{m}$ & $11.1 \pm 1.0$ & $11.7 \pm 1.2$ & $11.1 \pm 1.1$ & $10.5 \pm 0.8$ & $7.6 \pm 0.8^{*}$ & $9.9 \pm 0.5$ & $11.7 \pm 0.8$ & $12.1 \pm 0.9$ & $11.8 \pm 1.2$ \\
\hline $\mathrm{FCD}, \mathrm{cm} / \mathrm{cm}^{2}$ & $227 \pm 15$ & $242 \pm 13$ & $207 \pm 19$ & $228 \pm 15$ & $151 \pm 19 *$ & $149 \pm 17 *$ & $227 \pm 13$ & $241 \pm 10$ & $238 \pm 11$ \\
\hline Venule diameter, $\mu \mathrm{m}$ & $22.3 \pm 2$ & $23.7 \pm 1.9$ & $23.5 \pm 2.3$ & $26.1 \pm 3.6$ & $22.4 \pm 4.0$ & $22.3 \pm 5.1$ & $30.6 \pm 5.4$ & $27.3 \pm 4.2$ & $28.4 \pm 4.6$ \\
\hline Venular RBC velocity, $\mathrm{mm} / \mathrm{s}$ & $1.2 \pm 0.2$ & $1.3 \pm 0.1$ & $1.3 \pm 0.1$ & $1.7 \pm 0.2$ & $1.1 \pm 0.2 *$ & $1.4 \pm 0.4$ & $1.7 \pm 0.4$ & $1.6 \pm 0.3$ & $1.7 \pm 0.4$ \\
\hline
\end{tabular}

MAP = Mean arterial pressure. Diameter, FCD, and venular RBC velocity were assessed by computer-assisted image analysis. Data are given as means \pm SEM. p values calculated by Friedman's test and Dunn's follow-up testing. $p$ values $<0.05$ were considered significant. $*$ p $<$ 0.05 vs. baseline.

ing one to three postcapillary venules $(18-40 \mu \mathrm{m}$ in diameter) or arterioles $(8-14 \mu \mathrm{m}$ in diameter), as well as capillaries. By means of a computer-controlled stepping-motor-driven platform, identical vessel segments were reinvestigated with respect to the above-described parameters. For visualization of leukocytes, the fluorescent marker rhodamine $6 \mathrm{G}$ (Sigma) was injected intravenously as a single bolus of $0.15 \mathrm{mg} / \mathrm{kg}$ body weight immediately prior to each measurement. For labeling of the plasma, injection of the in vivo fluorescent plasma marker FITC-dextran (molecular mass $150 \mathrm{kD} ; 15 \mathrm{mg} / \mathrm{kg}$ body weight i.v.; Sigma, Oberhaching, Germany) was performed. The double-fluorescence technique allowed for subsequent investigation of vessel diameter, venular RBC velocity, functional capillary density (FCD), leukocyte-endothelial cell interaction, and macromolecular extravasation in identical vessel segments. Data analysis was performed off-line using a computer-assisted microcirculation analysis system (CAPImage/CAMAS, Dr. Zeintl, Heidelberg, Germany) [22].

\section{Microcirculatory Analysis}

The following microvasculatory parameters were assessed using intravital microscopy: FCD, diameter of arterioles and venules, and venular RBC velocity. Furthermore, leukocyte-endothelial cell interactions were determined by measuring the fraction of rolling leukocytes and the number of leukocytes adherent to the endothelium. FCD was assessed by image analysis (CAMS) as the length of RBCperfused capillaries per observation area (given as $\mathrm{cm} / \mathrm{cm}^{2}$ ). Rolling leukocytes were defined as cells which were rolling along the vessel visibly slower than the main bloodstream and loosely interacting with the endothelium. Differences in vessel diameter, blood flow velocity, and total number of leukocytes were corrected by examining the rolling fraction, given as the number of rolling leukocytes to the sum of rolling and all passing leukocytes through a defined vessel segment. Adherent leukocytes were defined as cells which remained attached to the endothelium for the entire 30-second observation period. Data are expressed as cells per square millimeter endothelial surface, which was calculated from the diameter and the length of the vessel segment $(200 \mu \mathrm{m})$.

\section{Statistical Analysis}

The data are expressed as means \pm SEM. Statistical significance was determined using repeated measurement ANOVA on ranks
(Friedman's test) and Dunn's follow-up testing. p values $<0.05$ were considered significant.

\section{Results}

Mean arterial blood pressure was not altered in any of the groups during the entire observation period (table 1). In the control group, there was no significant change in the microvascular parameters, as shown in table 1. In contrast, 10 min after injection of L-NAME the diameter of arterioles significantly decreased from $10.5 \pm 0.8$ to $7.6 \pm$ $0.8 \mu \mathrm{m}$ and remained decreased $(9.9 \pm 0.5 \mu \mathrm{m}) 60 \mathrm{~min}$ after injection. In the same group, the FCD was reduced from $228 \pm 15$ to $151 \pm 19 \mathrm{~cm} / \mathrm{cm}^{2}$ at $10 \mathrm{~min}$ and to $149 \pm$ $17 \mathrm{~cm} / \mathrm{cm}^{2} 60 \mathrm{~min}$ after injection. Furthermore, RBC velocity in postcapillary venules was reduced $10 \mathrm{~min}$ after injection of L-NAME from $1.75 \pm 0.21$ to $1.05 \pm 0.24$ $\mathrm{mm} / \mathrm{s}$. The fraction of rolling leukocytes and the number of cells adherent to the endothelium were increased $10 \mathrm{~min}$ after L-NAME injection from $0.20 \pm 0.03$ to $0.47 \pm 0.07$ and from $81 \pm 14$ to $236 \pm 41 \mathrm{~mm}^{-2}$, respectively (fig. 1 , 2). The number of adherent leukocytes remained elevated until $60 \mathrm{~min}$ after L-NAME injection $\left(361 \pm 105 \mathrm{~mm}^{-2}\right.$; fig. 2). Figure 3 shows two pictures of postcapillary venules of the synovium prior to the administration of L-NAME and 10 min after L-NAME injection, where large accumulation of leukocytes is visible. Furthermore, there was no significant change in the diameter of postcapillary venules in all groups (table 1). In contrast to the L-NAME group, no significant changes in the microvascular parameters were observed in the animals after L-NIL administration (table 1, fig. 1,2). 


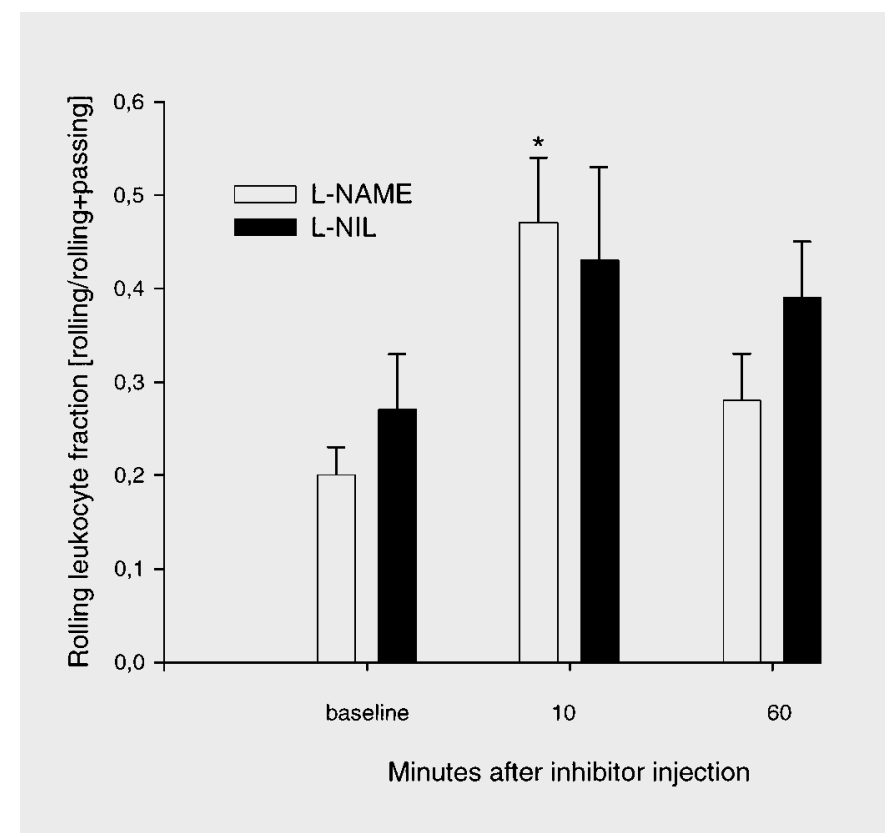

Fig. 1. Fraction of rolling leukocytes in postcapillary venules in the synovium of the mouse knee joint prior (baseline), and 10 and 60 min after intravenous injection of L-NAME and L-NIL. Data are given as means \pm SEM. ${ }^{*} \mathrm{p}<0.05$ vs. baseline.

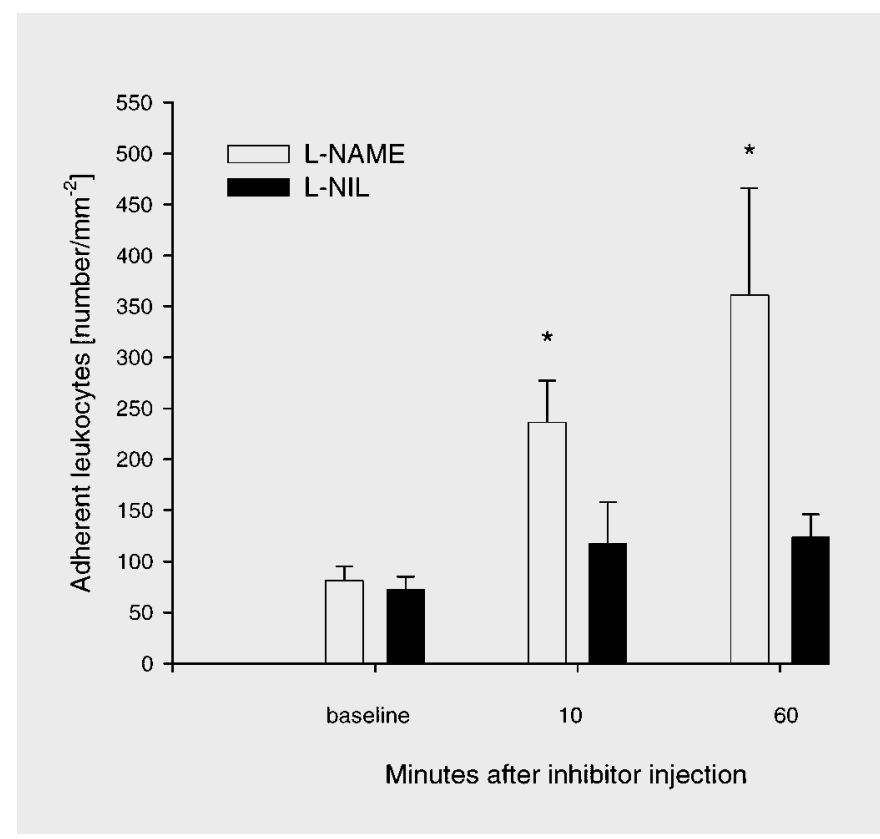

Fig. 2. Number of leukocytes adherent to the endothelium in postcapillary venules in the synovium of the mouse knee joint prior (baseline), and 10 and 60 min after intravenous injection of L-NAME and L-NIL. Data are given as means \pm SEM. $* \mathrm{p}<0.05$ vs. baseline.
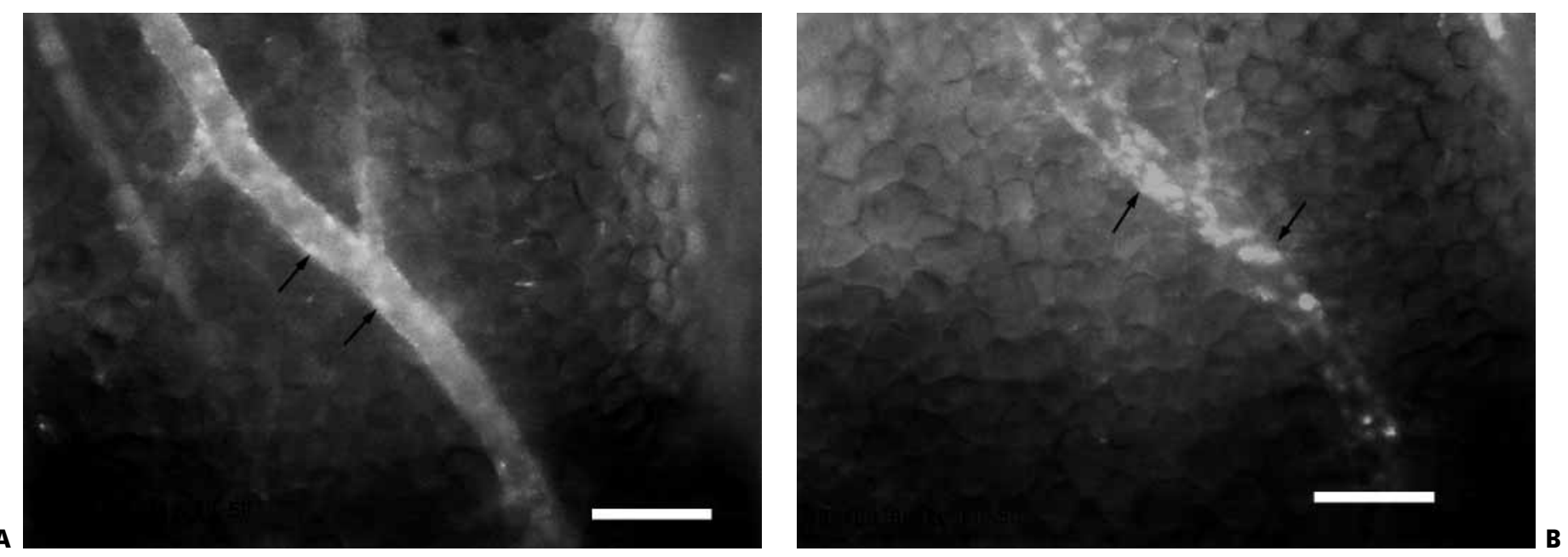

Fig. 3. Leukocyte-endothelial cell interaction in a postcapillary venule of synovial fat tissue visualized by intravital fluorescence microscopy after in vivo staining of leukocytes with rhodamine $6 \mathrm{G}(\times 307$, bars $=50 \mu \mathrm{m}$, arrows indicate leukocyte examples). A Prior to the administration of L-NAME only a few leukocytes can be seen. B Distinct accumulation of leukocytes occur 10 min after L-NAME injection. 


\section{Discussion}

The crucial role of iNOS-mediated NO production in chronic joint disease has been addressed by several studies, but it remained unclear whether this NO release is harmful or protective. Nonselective blocking of NOS has been shown to suppress the development of experimental arthritis in rats [8], although the therapeutic administration of both selective and nonselective iNOS inhibitors yielded contradictory results $[9,10]$. Moreover, little is known about the effects of NOS inhibition on the synovial microvasculature in vivo. Therefore, the aim of our study was to analyze the effects of selective versus nonselective inhibition of iNOS on the synovial microcirculation in vivo.

For this study, our recently described approach to the mouse knee joint using intravital microscopy of the synovial tissue was used [20]. The preparation for the quantitative analysis of the synovial microcirculation has been shown to be stable for at least $60 \mathrm{~min}$. The synovial tissue observed by this method is composed of the synovial cell layer, which is a thin cell layer, and the subsynovium. Within this tissue arterioles, capillaries, and venules can be analyzed quantitatively.

However, in studies investigating the effects of NOS inhibitors on the microcirculation in vivo, the mesentery but not the synovium was used [14, 18, 23]. In comparision to other soft tissues, the synovial microvasculature presents with a dense capillary network, a high RBC velocity, and a dense fenestration of the endothelium [20]. This difference in the microvasculature is presumably dud to the requirement of the synovium to nourish the avascular cartilage.

Our results demonstrate that inhibition of both NOS isoforms (cNOS and iNOS) by L-NAME has crucial effects on the synovial microcirculation. The increase in the fraction of rolling leukocytes $10 \mathrm{~min}$ after injection is in accordance with a study of Davenpeck et al. [13]. They observed a dose-dependent increase in leukocyte rolling after administration of L-NAME in the rat mesentery. It was suggested that this effect was most probably due to the presentation of P-selectin on endothelial cells and platelets after inhibition of NO production. P-selectin is an adhesion molecule that promotes leukocyte rolling and platelet adhesion. Nevertheless, after administration of NO donors, Kubes et al. [23] found that NO prevented leukocyte adhesion in ischemia-reperfusion of the rat mesentery, but failed to affect P-selectin-mediated leukocyte rolling. In another study, a significant enhancement of leukocyte adherence to the endothelium was observed

Synovial Microcirculation after NOS Inhibition in postcapillary venules in the rat mesentery after LNAME superfusion, which is in accordance with our results of an enhanced leukocyte accumulation in the synovium 10 and $60 \mathrm{~min}$ after L-NAME injection [14]. Several mechanisms may explain these results. Decrease in NO production results in a reduction of cGMP in endothelial cells, which leads to contraction of these cells and subsequent expansion of the junctions between adjacent endothelial cells, and platelet-leukocyte aggregation. Another effect of an attenuated NO release is the expression of adhesion molecules, such as CD11/CD18, VCAM1, and ICAM-1 [14, 24]. The mechanisms of L-NAMEinduced adhesion are still under debate. Recent studies have shown that NO can inhibit NF- $\mathrm{BB}$ activation and subsequent VCAM-1, ICAM-1, and E-selectin expression by increasing the expression and nuclear translocation of IкB- $\alpha[25,26]$. Finally, all these mechanisms may promote leukocyte adherence and subsequent emigration through the endothelium [14]. Another effect of L-NAME on the synovial microcirculation was a significant decrease in the arteriolar diameter and the FCD 10 and $60 \mathrm{~min}$ after administration. This is most likely due to inhibition of cNOS and subsequent arteriolar constriction, leading to a reduced perfusion of the synovial tissue. Further support of this explanation is the decrease in the RBC velocity in postcapillary venules throughout the entire observation period which was significant $10 \mathrm{~min}$ after L-NAME administration. In a recent study, local LNAME administration was found to reduced blood flow and enhance leukocyte adhesion via the expression of CD11/CD18 and its ligand ICAM-1 on the endothelium in rat skeletal muscle [24]. Furthermore, it has been shown by means of laser Doppler flowmetry that basal joint blood flow and vascular resistance were significantly reduced after L-NAME administration in the rabbit knee joint [27].

After selective iNOS inhibition with L-NIL, which has been shown to be a potent suppressor of iNOS in vitro and in vivo [19], we did not detect any significant alterations in the synovial microhemodynamics or leukocyte-endothelial cell interactions indicating that L-NIL does not inhibit cNOS. This would be the assumption for the therapeutic use of an iNOS inhibitor, which is suggested to be sufficient in experimental arthritis by a number of studies $[3,6,10]$. One supposed mechanism is that L-NIL suppresses intra-articular NO synthesis by the synovium and the cartilage, and thus protects the joint possible inflammatory and erosive effects of prolonged, excessive local production of NO. Another possibility is that iNOS inhibitors function as immunomodulators which downregulate 
the immune responses underlying the development of chronic joint inflammation [10].

In summary, we have shown that administration of LNAME leads to a decreased perfusion in the synovium as well as to an increased leukocyte adhesion. In contrast, L-NIL does neither alter the microhemodynamics nor the leukocyte-endothelial cell interaction in the mouse synovium significantly, indicating its potential use for therapeutic selective inhibition of iNOS in joint inflammation. Further investigations are necessary to evaluate whether selective iNOS inhibition has the potential to ameliorate acute and chronic joint diseases or whether it enhances the inflammatory reaction due to an enforced leukocyte adherence and emigration into synovial tissue. Such studies could help to understand the significance of the enhanced NO production in orthopedic disease such as chronic joint inflammation and aseptic loosening of joint prostheses.

\section{Acknowledgment}

The authors wish to thank to Dr. S. Massberg for many helpful comments and Dr. C. Birkenmeier for thoroughly reading the manuscript.

\section{References}

1 Stadler J, Stefanovic-Racic, Billiar RD, Curran RD, McIntyre LA, Georgescu HI, Simmons $\mathrm{RL}$, Evans $\mathrm{CH}$ : Articular chondrocytes synthetize nitric oxide in response to cytokines and lipopolysaccharide. J Immunol 1991;147: 3915-3921.

2 Stefanovic-Racic M, Stadler J, Evans CH: Nitric oxide and arthritis. Arthritis Rheum 1993; 36:1036-1044.

3 Cannon GW, Openshaw SJ, Hibbs JB, Hoidal JR, Hueckstaedt TP, Griffiths MM: Nitric oxide production during adjuvant-induced and collagen-induced arthritis. Arthritis Rheum 1996;39:1677-1684.

4 Grabowski PS, Wright PK, Van't Hof RJ, Helfrich MH, Oshshima H, Ralston SH: Immunolocalization of inducible nitric oxide synthase in synovium and cartilage in rheumatoid arthritis and osteoarthritis. Br J Rheumatol 1997;36:651-655.

5 Hilliquin P, Borderie D, Hernvann A, Menkes CJ, Ekindjian OG: Nitric oxide as S-nitrosoproteins in rheumatoid arthritis. Arthritis Rheum 1997;40:1512-1517.

6 Evans CH, Stefanovic-Racic M, Lancaster J: Nitric oxide and its role in orthopaedic disease. Clin Orthop 1995;312:275-294.

7 Veihelmann A, Brill T, Blobner M, Scheller I, Mayer B, Stadler J: Inhibition of nitric oxide improves detoxification in inflammatory liver dysfunction in vivo. Am J Physiol 1997;36: G530-G537.

8 McCartney-Francis N, Allen JB, Mizel DE, Albina JE, Xie Q, Nathan CF: Suppression of arthritis by an inhibitor of nitric oxide synthase. J Exp Med 1993;178:749-754.

9 Fletcher DS, Widmer WR, Luell S, Christen A, Orevillo C, Shah S, Visco D: Therapeutic administration of a selective inhibitor of nitric oxide synthase does not ameliorate the chronic inflammation and tissue damage associated with adjuvant-induced arthritis in rats. J Pharmacol Exp Ther 1998;284:714-721.
10 Stefanovic-Racic M, Meyers K, Meschter C, Coffey JW, Hoffman RA, Evans CH: Comparison of the nitric oxide synthase inhibitors methylarginine and aminoguanidine as prophylactic and therapeutic agents in rat adjuvant arthritis. J Rheumatol 1995;22:19221928.

11 Harris NR: Opposing effects of L-NAME on capillary filtration rate in the presence or absence of neutrophils. Am J Physiol 1997;273: G1320-G1325.

12 Baldwin AL, Thurston G, al Naemi H: Inhibition of nitrix oxide synthesis increases venular permeability and alters endothelial actin cytoskeleton. Am J Physiol 1998;274:H1176H1784.

13 Davenpeck KL, Gauthier TW, Lefer AM: Inhibition of endothelial-derived nitric oxide promotes P-selectin expression and actions in the rat microcirculation. Gastroenterology 1994; 107:1050-1058.

14 Kurose I, Kubes P, Wolf R, Anderson DC, Paulson J, Miyasaka M, Granger DN: Inhibition of nitric oxide production. Circ Res 1993; 73:164-171.

15 He P, Liu B, Curry FE: Effect of nitric oxide synthase inhibitors on endothelial $\left[\mathrm{Ca}^{2+}\right]_{\mathrm{i}}$ and microvessel permeability. Am J Physiol 1997; 272:H176-H185.

16 Rumbaut RE, McKay MK, Huxley VH: Capillary hydraulic conductivity is decreased by nitric oxide synthase inhibition. Am J Physiol 1995;268:H1856-H1861.

17 Hickey MJ, Sharkey KA, Sihota EG, Reinhardt $\mathrm{PH}$, MacMicking JD, Nathan C, Kubes P: Inducible nitric oxide synthase-deficient mice have enhanced leukocyte-endothelium interactions in endotoxemia. FASEB J 1997;11:955964.

18 McCafferty D-M, Mudgett JS, Swain MG, Kubes P: Inducible nitric oxide synthase plays a critical role in resolving intestinal inflammation. Gastroenterology 1997;112:1022-1027.
19 Stenger S, Thuring H, Rollinghoff M, Manning $\mathrm{P}$, Bogdan C: L-N6-(1-iminoethyl)-lysine potently inhibits inducible nitric oxide synthase and is superior to NG-monomethyl-arginine in vitro and in vivo. Eur J Pharmacol 1995;294: 703-712.

20 Veihelmann A, Szczesny G, Nolte D, Krombach, F, Refior HJ, Messmer K: A novel model for the study of synovial microcirculation in the mouse knee joint in vivo. Res Exp Med 1998; 198:43-54.

21 Harris AG, Hecht R, Peer F, Nolte D, Messmer $\mathrm{K}$ : An improved intravital microscopy system. Int J Microcirc 1997; 17:322-327.

22 Zeintl H, Sack F-U, Intaglietta M, Messmer K: Computer assisted leukocyte velocity measurements in intravital microscopy. Int J Microcirc 1989;8:293-302.

23 Kubes P, Kurose I, Granger DN: NO donors prevent integrin-induced leukocyte adhesion but not P-selectin-dependent rolling in postischemic venules. Am J Physiol 1994;267: H931-H937.

24 Mitchell DJ, Yu J, Tyml K: Local L-NAME decreases blood flow and increases leukocyte adhesion via CD18. Am J Physiol 1998;274: H1264-H1268.

25 Spiecker M, Peng HB, Liao JK: Inhibition of endothelial vascular cell adhesion molecule-1 expression by nitric oxide involves the induction and nuclear translocation of IkappaBalpha. J Biol Chem 1997;272:30969-30974.

26 Spiecker M, Darius H, Kaboth K, Hubner F, Liao JK: Differential regulation of endothelial cell adhesion molecule expression by nitric oxide donors and antioxidants. J Leukoc Biol 1998;63:732-739.

27 Najafipour H, Ferrell WR: Nitric oxide modulates sympathetic vasoconstriction and basal blood flow in normal and acutely inflamed rabbit knee joints. Exp Physiol 1993;78:615-624. 